\title{
ANÁLISE DE CRESCIMENTO DA CULTURA DA BATATA SUBMETIDA AO PARCELAMENTO DA ADUBAÇÃO NITROGENADA EM COBERTURA, EM UM LATOSSOLO VERMELHO-AMARELO
}

\author{
Growth analysis of potatoes (Solanum tuberosum L.) in response to nitrogen split \\ application in a red yellowish Latossol
}

Daniel Martins Barcelos', Anice Garcia², Vinicius Antonio Maciel Junior ${ }^{3}$

\begin{abstract}
RESUMO
O presente experimento foi instalado no Sítio Bela Vista, no município de São José da Bela Vista-SP, com o objetivo de verificar o efeito do parcelamento da cobertura com nitrogênio em características agronômicas da cultura da batata, cultivar Monalisa. A cultura foi adubada com $80 \mathrm{~kg} \cdot \mathrm{ha}^{-1}$ de N (sulfato de amônio) e demais nutrientes no momento do plantio. Foram estudados três tratamentos: tratamento 1 (T1), foi caracterizado pela aplicação em cobertura de $80 \mathrm{~kg}$.ha-1 de N, durante a amontoa; T2 caracterizouse pela aplicação de $40 \mathrm{~kg} \cdot \mathrm{ha}^{-1}$ de $\mathrm{N}$ na emergência das plantas e $40 \mathrm{~kg} \cdot \mathrm{ha}^{-1}$ de $\mathrm{N}$ durante a amontoa e T3 pela aplicação de $40 \mathrm{~kg} \cdot \mathrm{ha}^{-1}$ de $\mathrm{N}$ durante a amontoa e $40 \mathrm{~kg} \cdot \mathrm{ha}^{-1}$ de $\mathrm{N} 15$ após a amontoa. Os resultados mostraram os índices fisiológicos avaliados: produção de fitomassa seca de parte aérea, taxa de crescimento absoluto e taxa de crescimento relativo, apresentaram a mesma tendência, em todos os tratamentos e com relação 'a produção, não houve diferença significativa entre os tratamentos. A análise de parâmetros fisiológicos e de produção, em seu conjunto, permitem concluir que o cultivo da batata não foi afetado pelo parcelamento da dose de adubação do cobertura nitrogenada.
\end{abstract}

Termos para indexação: Análise de crescimento, batata, parcelamento, nitrogênio.

\section{ABSTRACT}

The experimental design was in full randomized block with 3 treatments and 4 replications. The treatment T1 was applied $80 \mathrm{~kg} . \mathrm{ha}-1$ of ammonium sulphate during the hilling; the treatment T2 was applied $40 \mathrm{~kg}$.ha- 1 of ammonium sulphate during the emergence and $40 \mathrm{~kg} \cdot \mathrm{ha}^{-1}$ during the hilling and the treatment T3 was applied $40 \mathrm{~kg} \cdot \mathrm{ha}^{-1}$ of ammonium sulphate during the hilling and $40 \mathrm{~kg} \cdot \mathrm{ha}^{-1}$ at 15 days after the hilling. The physiological parameters plant top dry matter, absolute growth rate and relative growth rate presented the same tendency, in all treatments, and crop yield did not differ significatively among the treatments. The analysis of physiological and production parameters, allow to conclude that the cultivation of the potato was not affected by the split application of nitrogen.

Index terms: Growth analysis, potatoes, split, nitrogen.

(Recebido para publicação em 1 de julho de 2004 e aprovado em 27 de junho de 2006)

\section{INTRODUÇÃO}

Embora a batata (Solanum tuberosum L.) responda à adição de nutrientes no solo, deve-se estar atento para não fornecer nutrientes em excesso, especialmente o nitrogênio. Excesso de nitrogênio causará nas plantas alto desenvolvimento vegetativo e atraso na tuberização (THOMPSON-JOHNS et al., 1998).

Alguns autores recomendam apenas uma aplicação em cobertura de nitrogênio e outros, embasados em estudos de perda de nitrogênio, sugerem o fracionamento da dose desse nutriente em mais de uma vez após o plantio constituindo-se numa alternativa interessante em solos de textura grosseira e sob irrigação (KLEINHENZ, 2001; ROSEN, 1991). Segundo Steiber \& Mahler (2003), a cultura de batata é uma das que requerem mais parcelamento do adubo nitrogenado.Os autores recomendam até cinco parcelamentos, pelo fato das raízes apresentarem-se superficiais e pela fácil perda de nitrogênio por lixiviação.

Com relação à maturidade, cultivares de batata podem ser divididos em três classes: precoces, ciclo normal e tardias, sendo que as precoces requerem altas taxas de nutrientes no início da estação de crescimento e respondem melhor à adubação pré-plantio, enquanto que as tardias usam nutrientes durante um período maior, nesse caso elas respondem positivamente ao parcelamento de nitrogênio (CANADA, 2003). Contudo, mesmo em cultivares precoces quando há necessidade de aplicação de altas doses de nitrogênio, o parcelamento é recomendável, uma vez que isto pode atrasar a tuberização

'Engenheiro Agrônomo - Faculdade Dr. Francisco Maeda/FAFRAM - Rod. Jeronimo Nunes Macedo, km 01 - Ituverava, SP.

${ }^{2}$ Professora, Dra., Faculdade de Agronomia Dr. Francisco Maeda/FAFRAM - Rod. Jeronimo Nunes Macedo, km 01 - Ituverava, SP -anice@feituverava.com.br ${ }^{3}$ Professor, MSc, Faculdade de Agronomia Dr. Francisco Maeda /FAFRAM - Rod. Jeronimo Nunes Macedo, km 01 - Ituverava, SP — viniciusmaciel@feituverava.com.br 
(DEPARTMENT OF AGRICULTURE AND FORESTRY, 2000).

Em geral, os produtores fazem uma adubação de cobertura junto com a operação de amontoa, operação na qual procede-se o chegamento de terra junto à planta, quando esta atinge a altura entre vinte a trinta centímetros, o que ocorre aos vinte a trinta dias após a emergência, período em que segundo Gargantini et al. (1963), é o período de máxima absorção de nitrogênio.

Os estudos sobre análise de crescimento de espécies vegetais possibilitam acompanhar o desenvolvimento das plantas como um todo e a contribuição dos diferentes órgãos no crescimento total, permitindo conhecer o seu funcionamento e suas estruturas (BENINCASA, 1988; LIEDGENS, 1993). A análise de crescimento é também, um método que tem sido utilizado com o objetivo primordial de gerar descrição clara do padrão de crescimento da planta ou de partes dela, permitindo comparações entre situações distintas, podendo ser aplicada às mais diversas modalidades de estudos (BEADLE, 1993; HUNT, 1978; LIEDGENS, 1993).

Beadle (1987) afirmou que a TCR é um parâmetro fundamental pra se avaliar a produção de matéria seca de um vegetal, podendo ser usado para comparar o desempenho de espécies vegetais ou um efeito de determinado tratamento. Além disso, Radford (1967) argumenta que esse índice fisiológico é o mais apropriado para comparar efeitos de diferentes manejos agronômicos, por ser relativo e não depender de pressuposições matemáticas. Assim, conduziu-se o presente trabalho para verificar os efeitos do parcelamento da cobertura nitrogenada em características de crescimento e produção da cultura da batata.

\section{MATERIAL E MÉTODOS}

O presente experimento foi instalado no Sítio Bela Vista, município de São José da Bela Vista-SP, situado a latitude $20^{\circ} 30^{\prime} 35^{\prime}$ " S e longitude $47^{\circ} 38^{\prime} 24^{\prime \prime}$ W e altitude de $730 \mathrm{~m}$, precipitação média da região de $1.400 \mathrm{~mm}$, em um Latossolo Vermelho Amarelo, o qual apresentou os seguintes resultados de análise química para a camada de 0-20 cm de profundidade: $\mathrm{pH}(\mathrm{CaCl} 2): 5,2 ;$ M.O.: $46 \mathrm{~g}^{-\mathrm{dm}^{-3}}$; P resina: $31 \mathrm{mg} \mathrm{dm}^{-3}$; K: $1,1 \mathrm{mmol}_{\mathrm{c} .} \mathrm{dm}^{-3}$; Ca: $32 \mathrm{mmol}_{\mathrm{c} .} \mathrm{dm}^{-3}$; $\mathrm{Mg}$ : $9 \mathrm{mmol} \mathrm{dm}^{-3}$; H+AL: $38 \mathrm{mmol}_{\mathrm{c}} \mathrm{dm}^{-3}$; SB: $42 \mathrm{mmol} \mathrm{dm}^{-3}$; $\mathrm{V}: 53 \%$; S: $65 \mathrm{mg} \cdot \mathrm{dm}^{-3} ; \mathrm{B}=0,25 \mathrm{mg} \cdot \mathrm{dm}^{-3} ; \mathrm{Cu}=5,6 \mathrm{mg}^{\mathrm{c}} \cdot \mathrm{dm}^{-3}$; $\mathrm{Fe}=19 \mathrm{mg} \cdot \mathrm{dm}^{-{ }^{3}} ; \mathrm{Mn}=5,8 \mathrm{mg} \cdot \mathrm{dm}^{-}{ }^{3}$ e $\mathrm{Zn}=1,0 \mathrm{mg} \cdot \mathrm{dm}^{-}{ }^{3}$, análise esta realizada no Laboratório de Análise Química de Solos da Faculdade "Dr. Francisco Maeda" (FAFRAM).
O plantio da batata, cultivar Monalisa, hábito de crescimento semi-ereta, acamamento regular, foi realizado em 20/05/2002. A batata-semente foi distribuída em sulcos, de aproximadamente $0,07 \mathrm{~m}$ de profundidade, manualmente, e, em em outro sulco lateral foi distribuído o adubo. A adubação de plantio, baseada na análise de solo, e seguindo recomendações técnicas Instituto Agronômico (1998), foi comum a todas às parcelas e correspondeu a $80 \mathrm{~kg}$.ha-1 de nitrogênio (sulfato de amônio), $200 \mathrm{~kg} \cdot \mathrm{ha}^{-1}$ de $\mathrm{P}_{2} \mathrm{O}_{5}$ (superfosfato simples), $150 \mathrm{~kg} \cdot \mathrm{ha}^{-1}$ de $\mathrm{K}_{2} \mathrm{O}$ (cloreto de potássio) e $2 \mathrm{~kg} \cdot$ ha $^{-1}$ de boro (bórax). Para a adubação de cobertura foram utilizados $80 \mathrm{~kg} \cdot \mathrm{ha}^{-1}$ de nitrogênio (sulfato de amônio) em cada tratamento.

O delineamento experimental adotado foi o de blocos ao acaso, com quatro repetições e três tratamentos. A unidade experimental foi constituída por quatro linhas de 3,6 m de comprimento, espaçadas entre si de 0,9 m; cada parcela ocupou a área útil de $13 \mathrm{~m}^{2}$.

Os tratamentos foram:

T1: aplicação em cobertura de $80 \mathrm{~kg} \cdot \mathrm{ha}^{-1}$ de nitrogênio durante a amontoa, que ocorreu aos 16 dias após a emergência;

T2: aplicação de $40 \mathrm{~kg} \cdot \mathrm{ha}^{-1}$ de nitrogênio na emergência das plantas e $40 \mathrm{~kg} \cdot \mathrm{ha}^{-1}$ de nitrogênio durante a amontoa;

T3: aplicação de $40 \mathrm{~kg} \cdot \mathrm{ha}^{-1}$ de nitrogênio durante a amontoa e $40 \mathrm{~kg} \cdot \mathrm{ha}^{-1}$ de nitrogênio, 15 dias após a amontoa.

A “amontoa" foi efetuada 16 dias após a emergência, com a cultura tendo entre $20-30 \mathrm{~cm}$. Por ocasião da amontoa foi realizada a aplicação de inseticida (Carbofuran), na dose de $30 \mathrm{~kg} \cdot \mathrm{ha}^{-1}$.

Dezessete dias após o plantio realizou-se o controle de ervas daninhas, antes da emergência da cultura, utilizando-se herbicida de contato ( Paraquat ), na dose de 0,5 L.ha-1 .

O controle fitossanitário foi realizado por pulverizações preventivas semanais, com fungicida (Ditiocarbamato), na dose de $3 \mathrm{~kg} \cdot \mathrm{ha}^{-1}$ e inseticida (Metamidophos), na dose de 0,5L.ha-1 .

Durante a condução do experimento ocorreu somente uma precipitação pluviométrica de $30 \mathrm{~mm}$, dois dias após o plantio. A cultura foi irrigada semanalmente, por aspersão.

Aos 37, 52 e 67 dias após a emergência (DAE) foi avaliada a produção de fitomassa seca de parte aérea de plantas. Em cada parcela foram coletadas, aleatoriamente, duas plantas úteis, as quais foram colocadas para secar em estufa a $70^{\circ} \mathrm{C}$ até peso constante, e posteriormente determinada a fitomassa seca. A partir da fitomassa seca

Ciênc. agrotec., Lavras, v. 31, n. 1, p. 21-27, jan./fev., 2007 
determinou-se os parâmetros para análise de crescimento: taxa de crescimento absoluto e a de crescimento relativo, como seguem:

$$
\begin{gathered}
\mathrm{TCA}=(\mathrm{P} 2-\mathrm{P} 1) /(\mathrm{t} 2-\mathrm{t} 1) \\
\mathrm{TCR}=(\mathrm{LnP} 2-\mathrm{LnP} 1) /(\mathrm{t} 2-\mathrm{t} 1)
\end{gathered}
$$

em que, TCA É a taxa de crescimento absoluto; TCR é a taxa de crescimento relativo; $\mathrm{P}$ é o peso total da matéria seca da planta; t é o intervalo de tempo entre duas amostragens, e 1 e 2 referem-se a duas amostragens sucessivas (BEADLE, 1987; BENINCASA, 1988; CAUSTON \& VENUS, 1981; RADFORD, 1967).

Realizou-se a colheita quando os tubérculos atingiram a maturidade fisiológica, o que ocorreu aos 107 dias após a emergencia. A colheita foi semi-mecanizada, o arranquio feito por meio mecanizado e os tubérculos foram coletados manualmente, colhendo-os nas duas linhas centrais, eliminando os tubérculos da primeira e última planta dessas linhas.

Avaliou-se a produtividade pela colheita de todos os tubérculos contidos nas duas linhas centrais da parcelas. Pela proporção entre massa total e área útil da parcela experimental, obteve-se a produtividade total $\left(\mathrm{kg} \cdot \mathrm{ha}^{-1}\right)$.
Para avaliação da fitomassa seca em cada um dos tratamentos foram utilizadas regressões que mostrassem o comportamento da fitomassa no tempo.

\section{RESULTADOS E DISCUSSÃO}

Na Figura 1, encontram-se os dados referentes à avaliação de matéria seca de parte aérea das plantas de batata. Pode-se observar que os três tratamentos não diferiram, significativamente, entre si (teste de Tukey a 5\% de probabilidade), para este índice fisiológico.

O comportamento da fitomassa em relação ao tempo é o esperado, visto que a variação da fitomassa seca está diretamente relacionada com a área foliar da cultura e a variação temporal desta, em geral, aumenta até um máximo, que ocorre entre 61 a 70 dias (ROBLES, 2003), em que permanece por algum tempo, decrescendo em seguida, devido à senescência das folhas velhas. Como a fotossíntese depende da área foliar, o rendimento da cultura será maior quanto mais rápido a planta atingir o índice de área foliar máximo e quanto mais tempo a área foliar permanecer ativa (PEREIRA \& MACHADO, 1987).

Nas Figuras 2, 3 e 4 mostram-se a curva de regressão para as três avaliações realizadas para os tratamento $\mathrm{T} 1, \mathrm{~T} 2 \mathrm{e} \mathrm{T} 3$, respectivamente.

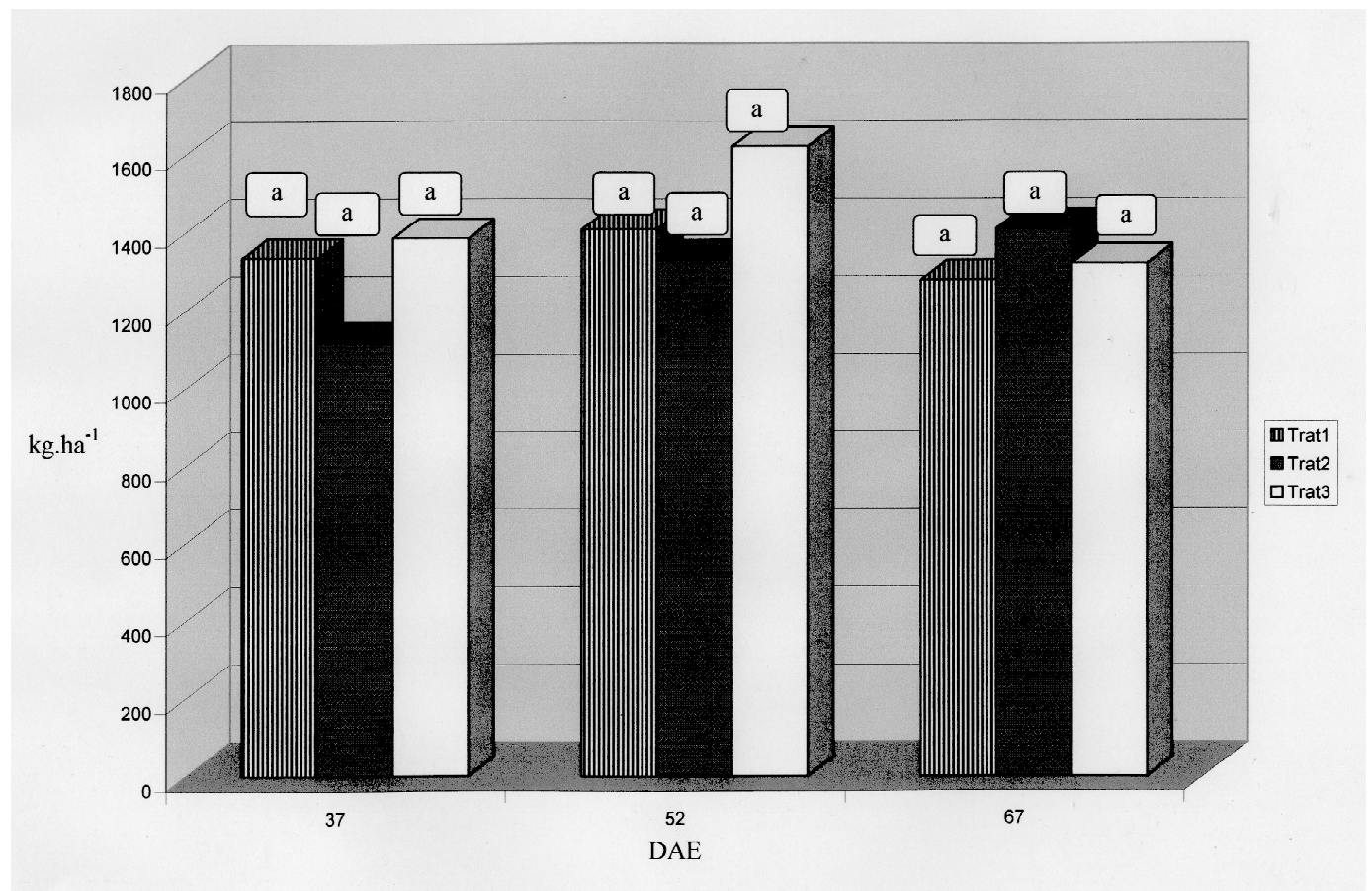

FIGURA 1 - Variação de fitomassa seca de parte aérea de plantas de batata - São José da Bela Vista - SP. 


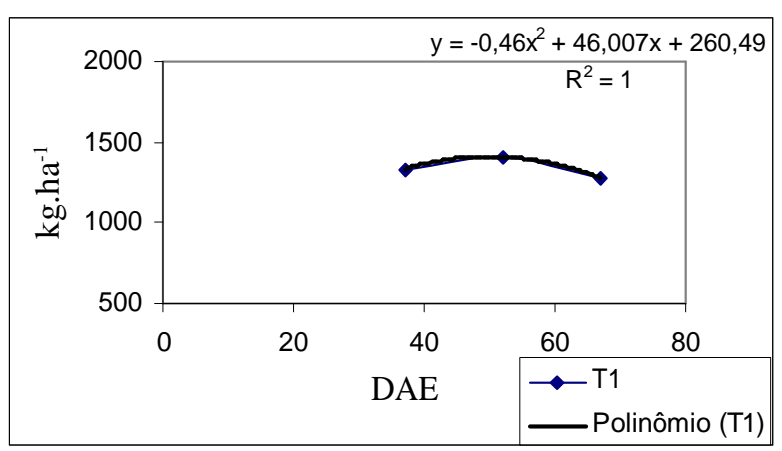

FIGURA 2 - Fitomassa seca total em parte aérea de plantas de batata ao longo do ciclo $\left(\mathrm{kg} \cdot \mathrm{ha}^{-1}\right)$, para o tratamento T1. - São José da Bela Vista - SP.

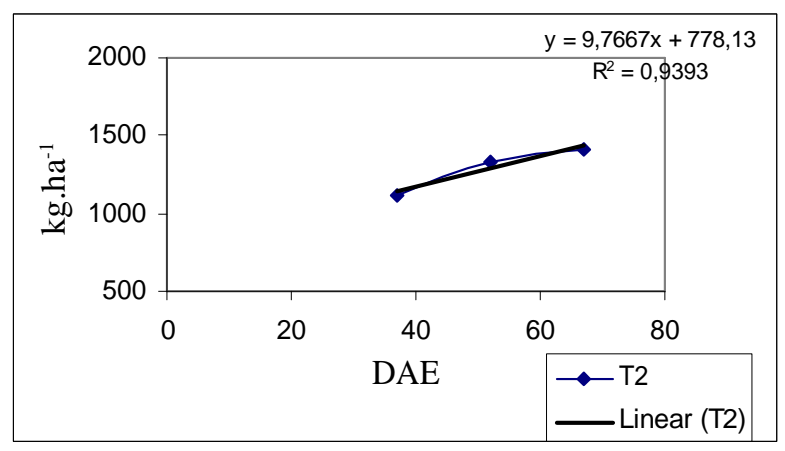

FIGURA 3 - Fitomassa seca total em parte aérea de plantas de batata ao longo do ciclo $\left(\mathrm{kg} \cdot \mathrm{ha}^{-1}\right)$, para o tratamento T2- São José da Bela Vista - SP.

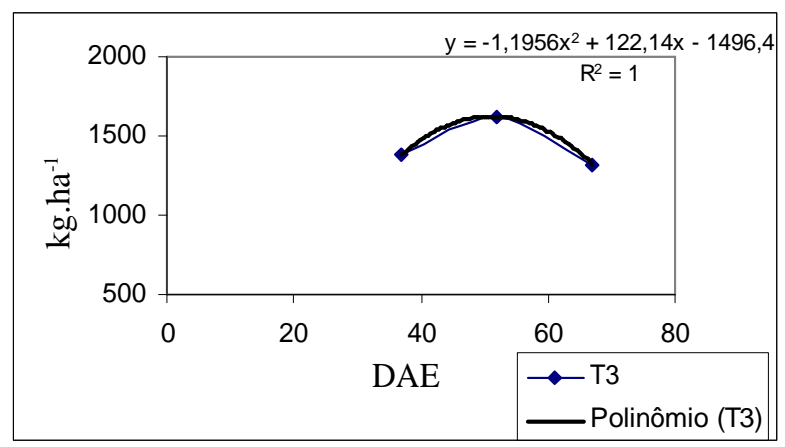

FIGURA 4 - Fitomassa seca total em parte aérea de plantas de batata ao longo do ciclo $\left(\mathrm{kg} \cdot \mathrm{ha}^{-1}\right)$, para o tratamento T3. - São José da Bela Vista - SP.
Os tratamentos 1 e 3 apresentaram curvas que ajustaram-se ao modelo quadrático de regressão para matéria seca de parte aérea, com $\mathrm{R}^{2}=1$, indicando que $100 \%$ dos dados obtidos são ajustados pela regressão. Pela equação quadrática observa-se que até determinado período da cultura no campo há um aumento da matéria seca, depois a tendência é diminuir. Embora tenha sido usado somente três pontos ( 3 avaliações) para a obtenção das regressões, o que pode ser cosiderado pouco para a obtenção de regressões, o comportamento observado para fitomassa seca de plantas é normalmente esse, isto é, há um aumento e posterior redução no final do ciclo.

O tratamento 2 (Figura 3 ) apresentou curva que se ajusta ao modelo linear, com $\mathrm{R}^{2}=0.93$, indicando que 93\% dos dados obtidos são ajustados pela regressão. Pela equação linear observa-se que esse tratamento até a última avaliação feita ainda não havia começado a diminuir a matéria seca, o que deve ter ocorrido após a última avaliação.

Com base nas equações obtidas, em que $\mathrm{X}$ é o número de dias após a emergência e $\mathrm{Y}$ é a quantidade de fitomassa seca total de parte aérea, pode-se estimar a quantidade de fitomassa seca total de parte aérea em qualquer momento durante o experimento, o que nos permite estimar diariamente o acréscimo e/ou diminuição da matéria seca das plantas e conseqüentemente suas taxas de crescimento absoluto e relativo.

A taxa de crescimento absoluto (TCA) (Figura 5) pode ser usada para analisar a velocidade média de crescimento ao longo do período de observação. A tendência apresentada pelos tratamentos, em relação a esse índice fisiológico, foi a mesma, isto é, houve um declínio da TCA a partir da primeira avaliação. A TCA máxima foi de 36, 30 e 37 g.dia $^{-1}$, para os tratamentos T1, T2 e T3, respectivamente.

As curvas da taxa de crescimento relativo (TCR) em razão dos DAE da cultura da batata, e submetidas a diferentes épocas de adubação de cobertura são mostradas na Figura 6. A TCR reflete o aumento da matéria orgânica seca, em gramas, das plantas, num período de tempo, sendo função do tamanho inicial, ou seja, do material pré-existente.

Assim, percebe-se uma fase inicial de rápido acúmulo de material, seguida de uma com menor incremento. Esse comportamento de TCR é amplamente reportado na literatura, como em Aguiar Neto et al. (2000) e Benincasa (1988), pode ser explicada pelo aumento da competição intraespecífica pelos principais fatores ambientais responsáveis pelo crescimento (GAVA et al., 2001).

Ciênc. agrotec., Lavras, v. 31, n. 1, p. 21-27, jan./fev., 2007 


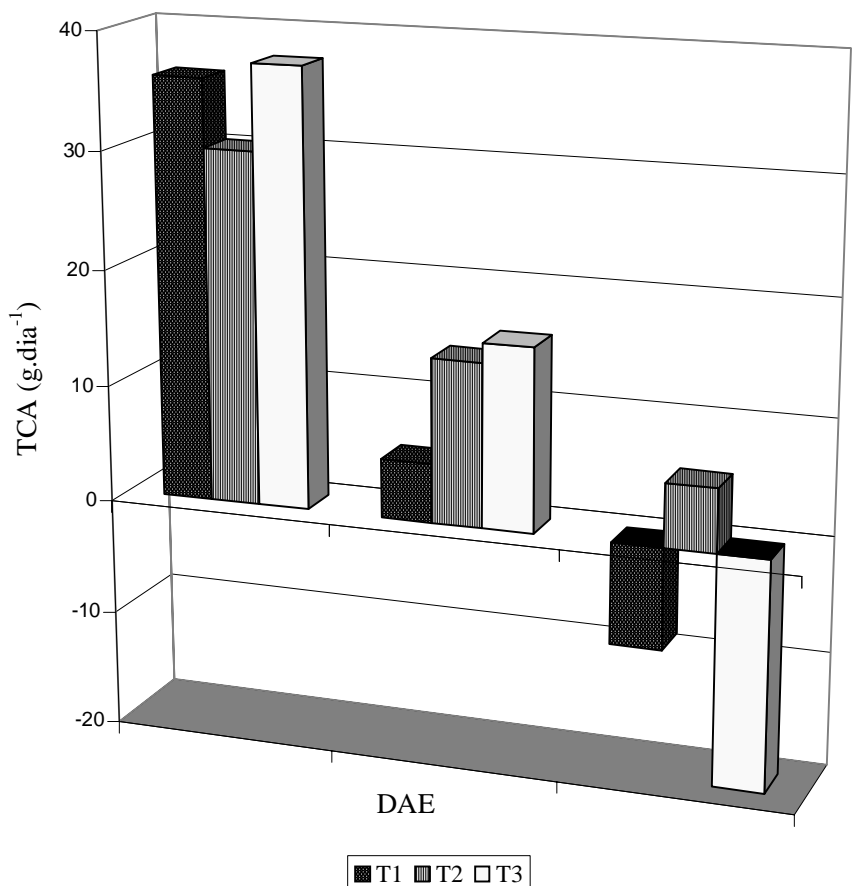

FIGURA 5 - Taxa de crescimento absoluto para plantas de batata submetida a diferentes parcelamentos da adubação de nitrogenada em cobertura.São José da Bela Vista - SP.

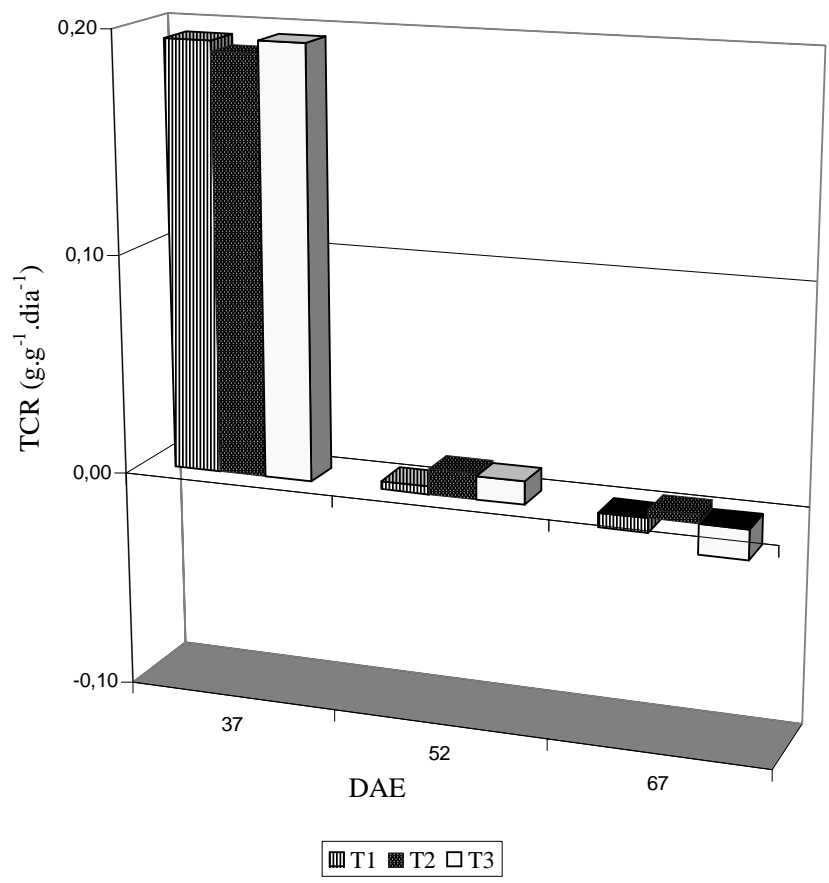

FIGURA 6 - Taxa de crescimento relativo para plantas de batata submetidas a diferentes parcelamentos da adubação nitrogenada em cobertura. São José da Bela Vista - SP.

Ciênc. agrotec., Lavras, v. 31, n. 1, p. 21-27, jan./fev., 2007 
O declínio dessa taxa de crescimento, portanto, é esperada, conforme a planta vai atingindo a maturidade. Na primeira avaliação os valores médios de TCR foram de $0,20 \mathrm{~g} \cdot \mathrm{g}^{-1} \cdot \mathrm{dia}^{-1} \mathrm{~A}$ taxa de crescimento relativo (TCR) tendeu a decrescer com a evolução do crescimento após 50 DAE.

Aguiar Neto et al. (2000) observam que em relação ao tempo, verifica-se que a tendência geral de TCR é a redução com o desenvolvimento do ciclo da cultura da batata. Esses autores, estudando o comportamento da cultura sob estresse hídrico, observaram de um modo geral, uma fase inicial de rápido acúmulo de material, seguida de uma com menor incremento, com um posterior período de valores próximos a zero.

Os três tratamentos praticamente não apresentaram diferenças quanto a este índice fisiológico.

Pela Tabela 1, apresentam-se os dados de produção de tubérculos de batata ao final do experimento.

Observa-se pela Tabela 1, que não houve diferença significativa entre os tratamentos estudados. De acordo com os dados obtidos, estima-se produção média de 35,1; 35,2 e 38,0 t/ha para os tratamentos 1, 2 e 3 , respectivamente, o que corroboram a produção para essa cultivar obtida por Nunes (2002). Os valores ligeiramente superiores de produção apresentados no tratamento 3, confirmam os dados de TCA e TCR, que também foram superiores para esse tratamento.

TABELA 1 - Produção de tubérculos de batata por parcela experimental em kg (São José da Bela Vista - SP).

\begin{tabular}{c|c}
\hline Tratamento & Produção $\left(\mathbf{k g} / \mathbf{6 , 5} \mathbf{m}^{\mathbf{- 2}}\right)$ \\
\hline 1 & $22,77 \mathrm{a}$ \\
\hline 2 & $22,80 \mathrm{a}$ \\
\hline 3 & $24,62 \mathrm{a}$ \\
\hline
\end{tabular}

\section{$\mathrm{dms}=4,1$}

$\mathrm{CV}=8,86 \%$

Obs: médias seguidas de letras iguais não diferem significativamente entre si ao nível de $5 \%$.

\section{CONCLUSÃO}

A análise de parâmetros fisiológicos e de produção, em seu conjunto, permitem concluir que o cultivo da batata não foi afetado pela parcelamento da dose de adubação do cobertura nitrogenada.

\section{REFERÊNCIAS BIBLIOGRÁFICAS}

AGUIAR NETTO, A. O.; RODRIGUES, J. D.; PINHO, S. Z. Análise de crescimento na cultura da batata submetida a diferentes laminas de irrigação. Pesquisa Agropecuária Brasileira, Brasília, v. 35, n. 5, p. 901-907, 2000.
BEADLE, C. L. Growth analysis. In: HALL, D. O.; SCURLOCK, J. M. O.; BOLHR-NORDENKAMPF, H. R.; LEEGOOD, R. C.; LONG, S. P. (Eds.). Photosynthesis and production in a changing environment: a field and laboratory manual. London: Chapman \& Hall, 1993. p. 36-46.

BEADLE, C. L. Plant-growth analysis. In: COOMBS, J.; HALL, D. O. (Eds.). Techniques in bioproductivity and photosynthesis. Oxford: Pergamon, 1987. p. 20-25.

BENINCASA, M. M. P. Análise de crescimento de plantas. Jaboticabal: FUNEP, 1988. 43 p.

CANADA. Irrigation Diversification Centre. Potato cultivar specific fertility mahagenment. 2001. Disponível em: $\langle\mathrm{http} /$ hwww.agr.ca/pfra/sldegene.htm'>. Acesso em: 28 abr. 2003.

CAUSTON, D. R.; VENUS, J. C. The biometry of plant growth. London: Arnold, 1981. 307 p.

DEPARTMENT OF AGRICULTURE AND FORESTRY.Split nitrogen applications for potatoes. Disponível em: <http:/ i/www.gov.pe.ca/af/soilfeed/fact_split.php $3 \overline{3}$. Acesso em: 3 fev. 2000.

GARGANTINI, B. G.; GALLO, J. R.; NÓBREGA, S. A. Absorção de nutrientes pela batatinha. Bragantia, Campinas, v. 22, p. 267-289, 1963.

GAVA, G. J. C.; TRIVELIN, P. C. O.; OLIVEIRA, M. W. Growth and accumulation of nitrogen by sugarcane cultivated in soil covered with cane trash. Pesquisa Agropecuária Brasileira, Brasília, v. 36, n. 11, p. 13471354, nov. 2001.

HUNT, R. Plant growth analysis. London: Arnold, 1978. 67 p.

INSTITUTO AGRONÔMICO. Instruções agrícolas para as principais culturas econômicas. 6. ed. Campinas, 1998. 396 p. (Boletim, 200).

KLEINHENZ, M. Potatoes growing tips and news from the world of research. The Tuber Times, Ohio, v. 2, n. 1, 2001.

LIEDGENS, M. M. Modelos numéricos para a descrição do crescimento da planta de soja (Glycine Max L. Merrill, cultivar IAC-15) em condições sazonais diferenciadas. 1993. 101 f. Dissertação (Mestrado) - Universidade Estadual de Campinas, Campinas, 1993. 
NUNES, M. U. C. Yield and phytosanitary problems of potato cultivars in Sergipe, Brazil. Horticultura Brasileira, Brasília, v. 20, n. 3, p. 424-427, 2002.

PEREIRA, A. R.; MACHADO, E. C. Análise quantitativa do crescimento de comunidade vegetal. Campinas: Instituto Agronômico, 1987. 33 p. (Boletim técnico, 114).

RADFORD, P. J. Growth analysis formulae: their use and abuse. Crop Science, Madison, v. 7, n. 3, p. 171-175, 1967.

ROBLES, W. G. R. Dióxido de carbono via irrigação em batateira (Solanum tuberosum L.) sob condições de campo.
2003. 160 f. Tese (Doutorado) - Escola Superior de Agricultura “Luiz de Queiroz”, Universidade de São Paulo, Piracicaba, 2003.

ROSEN, C. J. Potato fertilization on irrigated soils. Minnesota: University of Minnesota, 1991. 7 p.

STEIBER, T. D.; MAHLER, R. L. Cropping practices survey: fertilizer results. Idaho: University of Idaho, 2003. 3 p.

THOMPSON-JOHN, A. et al. Potato production in the home garden. Idaho: University of Idaho, 1998. 